\title{
HARP TRACKING REFINEMENT USING SEEDED REGION GROWING
}

\author{
Xiaofeng Liu ${ }^{1}$, Emi Murano $^{3}$, Maureen Stone $^{3}$, and Jerry L. Prince ${ }^{1,2}$ \\ ${ }^{1}$ Computer Science, The Johns Hopkins University, Baltimore, MD, 21218 \\ ${ }^{2}$ Electrical and Computer Engineering, The Johns Hopkins University, Baltimore, MD, 21218 \\ ${ }^{3}$ Biomedical Sciences and Orthodontics , University of Maryland Dental School, Baltimore, MD, 21201
}

\begin{abstract}
Tagged magnetic resonance (MR) imaging makes it possible to image the motion of tissues such as the muscles found in the heart and tongue. The harmonic phase (HARP) method largely automates the process of tracking points within tagged MR images. It works by finding spatial points in successive images that retain the same two harmonic phase values throughout the entire image sequence. Given a set of tracked points, many interesting and useful motion properties such as regional displacement or rotation, elongation, strain, and twist, can be computed. When there is a large motion between successive image frames, HARP tracking can fail, and this results in mistracked points and erroneous motion estimates. In this paper, we present a novel HARP refinement method based on seeded region growing that addresses this problem. Starting from a given seed point which is determined by the user to be correctly tracked throughout the entire sequence, this method can reliably track the motion of the whole tissue. A novel cost function is used in the region growing to assure that points that can be most reliably tracked are tracked first. Experimental results on tagged MR images of the tongue demonstrate very reliable tracking.

INDEX TERMS - MR tagging, HARP, motion tracking, region growing
\end{abstract}

\section{INTRODUCTION}

Magnetic resonance (MR) tagging creates patterns of magnetic spin systems within the tissue, yielding images that carry information about motion within homogeneous tissues. This information complements traditional anatomical images, which carry information about motion only at the boundaries of tissues. Detailed information about the motion of tissues such as the heart and tongue throughout the muscles can be imaged in this way. Displacement, velocity, rotation, elongation, strain, and twist are just some of the quantities that be computed from this data. The harmonic phase (HARP) method $[1,2]$ processes tagged MR images, enabling the automatic computation of these quantities. The HARP method has been

This research was supported by NIH grants R01 HL047405 and R01 DC001758 successfully applied in both the heart [1] and the tongue [3], and has proved to be useful for both scientific and clinical applications.

Two-dimensional (2D) in-plane motion tracking is an important part of the HARP method because other quantities are often computed using these tracking results. HARP tracking (explained below) implicitly assumes that tissue points do not move much from one time frame to the next. If the tissue moves too fast, the temporal resolution is too low, or the MR tag parameters are selected incorrectly, this assumption is violated, and HARP tracking will fail. Although such failures are relatively rare in typical well-designed applications, careful scientific studies and robust clinical applications require that the user manually identify and correct mistracked points. This can be very time-consuming, to the point where large research studies take too much time and clinical throughput is too low. In our research on tongue motion, which motivated this particular research result, there are some utterances in which parts of the tongue move quite fast relative to the temporal resolution of the scan, causing inevitable HARP tracking errors. Efforts to track a very large number of points in the tongue $[4,5]$ thereby become extremely time consuming, as manual correction is routinely required.

There have been some previous efforts to identify and automatically correct mistracked points. Khalifa et al. [6] recently proposed a HARP tracking error correction method on cardiac tagged MR images. This method used an active contour model to correct the tracking error on a predefined circular mesh model. The approach is limited to the circular geometry and not easily generalized for applications in the tongue. In [1], the idea of HARP refinement - which we expand herein - was mentioned, but it was not considered to be computationally feasible [7]. Refinement was also developed for circular geometries, but we show herein, it is readily generalizable to arbitrary regions, even whole images.

In this paper, we proposed a refinement method for HARP tracking that is based on seeded region growing [8]. It can reliably track every point (pixel) inside a given tissue (or even the whole image) and does not require a circular geometry or organized mesh of points defined on the region. This approach, which is computationally fast, extends the original HARP tracking method making Lagrangian strain computa- 


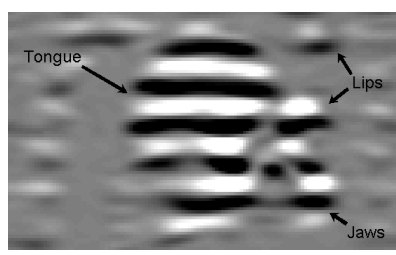

(a)

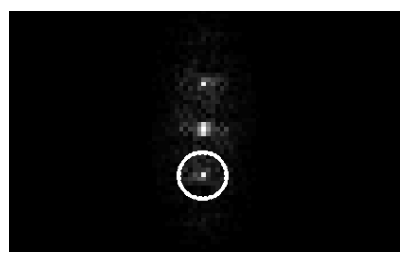

(b)
Fig. 1. (a) A tagged mid-sagittal MR image of the lower-half of the head and (b) the magnitude of its Fourier transform.

tions between arbitrary points feasible. Experiment results on MR tagged images of the tongue are used to demonstrate the effectiveness of this method.

\section{METHOD}

\subsection{HARP method and motion tracking}

The Fourier transform of a SPAMM-tagged MR image has two harmonic peaks, as shown in Fig. 1. In the HARP method, a bandpass filter is used to extract just one of the harmonic peaks, and its inverse Fourier transform is given by [2]

$$
I(\mathbf{x}, t)=D(\mathbf{x}, t) e^{j \phi(\mathbf{x}, t)},
$$

where $D(\mathbf{x}, t)$ is the harmonic magnitude image, and $\phi(\mathbf{x}, t)$ is the harmonic phase (HARP) image. The magnitude image reflects the tissue anatomy, and the HARP image contains the tissue motion information.

The harmonic phase is a material property of tagged tissue. Thus, for a material point $\mathbf{x}=\mathbf{x}(t)$, and $\mathbf{x}(0)=x_{0}$, its phase value $\phi(\mathbf{x}(t))=\phi\left(\mathbf{x}_{0}\right)$ does not change as the point moves. The HARP value is the principal value of the corresponding phase value

$$
\alpha_{(1,2)}(\mathbf{x}, t)=W\left(\phi_{(1,2)}(\mathbf{x}, t)\right)
$$

where $W(\cdot)$ is the wrapping function defined as:

$$
W(\phi(\mathbf{x}, t))=\bmod (\phi+\pi, 2 \pi)-\pi
$$

such that $\alpha_{(1,2)}(\mathbf{x}, t) \in[-\pi, \pi)$, and $(1,2)$ gives one of the two tag orientations.

It is observed that the HARP value is also a material property and therefore does not change as $\mathbf{x}$ moves either. This is called phase invariance property and is the basis of HARP motion tracking. For 2D tracking, two tag orientations are required to track the apparent motion of a material point. The HARP tracking method is described in detail in [1]. Briefly, when given the position of a material point $\mathrm{x}$ at some time frame $t_{i}$, the HARP method searches its neighborhood at next time frame $t_{i+1}$ in both tag orientations to find the closest point $\mathbf{x}\left(t_{i+1}\right)$ such that

$$
\alpha_{(1,2)}\left(\mathbf{x}\left(t_{i}\right), t_{i}\right)=\alpha_{(1,2)}\left(\mathbf{x}\left(t_{i+1}\right), t_{i+1}\right)
$$

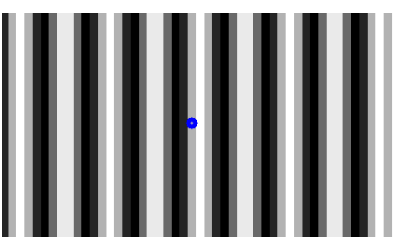

(a)

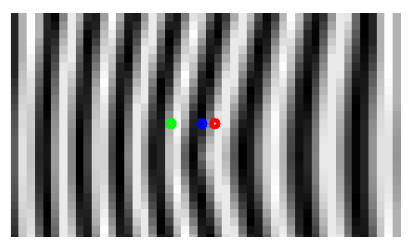

(b)
Fig. 2. This example illustrates when HARP tracking fails. (a) is the image at first time frame, and (b) is the image at the second time frame. In both images, the blue dot is the point being tracked. In (b), the green dot is where it actually moves, and the red dot is the result of HARP tracking. The HARP tracking fails because the red dot is the closest to the blue dot that has the same phase value at the second time frame.

If the tissue moves more than half of the tag separation between two successive time frames, HARP tracking will fail. To be clear, the HARP tracking algorithm will converge in all cases, but in the case of large motion, it will converge to a point having the same pair of HARP values but is one or more tag periods away from the true corresponding point. Fig. 2 shows such an example that 1D HARP tracking fails for large motion.

\subsection{HARP Refinement}

It is observed that the motion field within the tissue is smooth - e.g., the displacements of neighboring tissue points are similar. For two neighboring 2D points $\mathbf{x}\left(t_{0}\right), \mathbf{y}\left(t_{0}\right)$ at time frame $t_{0}$, and at time $t$ they move to $\mathbf{x}(t)$ and $\mathbf{y}(t)$, then the difference between their displacements

$$
\left.\Delta\left(\mathbf{x}, \mathbf{y}, t_{0}, t\right)\right)=\left|\left(\mathbf{x}(t)-\mathbf{x}\left(t_{0}\right)\right)-\left(\mathbf{y}(t)-\mathbf{y}\left(t_{0}\right)\right)\right|
$$

is small. So if $\mathbf{x}(t)$ can be tracked correctly from $\mathbf{x}\left(t_{0}\right)$, a good estimation of $\mathbf{y}(t)$ is

$$
\mathbf{y}^{\prime}(t)=\mathbf{y}\left(t_{0}\right)+\left(\mathbf{x}(t)-\mathbf{x}\left(t_{0}\right)\right)
$$

Therefore $\mathbf{y}^{\prime}(t)$ can be used as the starting point when tracking $\mathbf{y}\left(t_{0}\right)$ to prevent HARP tracking from failing.

There is usually some part of the tissue that has relatively small motion and can be tracked correctly using HARP over all time frames. For example, the bottom part of the tongue is relatively stationary in speech. Hence, it is easy to manually identify a few seed points where the traditional HARP tracking succeeds.

Based on the facts above we propose a new HARP refinement method that uses seeded region growing. Our algorithm starts with one or more manually identified seed points that can be correctly tracked over all time frames using traditional HARP tracking. From every time frame to the next, the neighbor points of the tracked seeds are identified and stored in a sequentially sorted list (SSL). At each iteration, the first point 
in the SSL is taken off the list and tracked, and its neighbors are inserted into the SSL in a way described below. The process is repeated until the list is empty. Since the tracking between different time frames is done independently, we consider only the tracking from time $t_{i}$ to $t_{i+1}$ in the following.

The SSL is maintained throughout the algorithm. The points in the SSL are called boundary points. Each entry on the list contains the following information: the point's 2D coordinate $\mathbf{y}\left(t_{i}\right)$ at time $t_{i}$, an initial estimate of its location in the next time frame $\mathbf{y}^{\prime}\left(t_{i+1}\right)$, and a cost value $c\left(\mathbf{y}\left(t_{i}\right)\right)$ (defined below). $\mathbf{y}^{\prime}\left(t_{i+1}\right)$ is computed using (6), assuming that $\mathbf{y}\left(t_{i}\right)$ has the same displacement as its tracked neighbor when it is inserted. The list is sorted in the ascending order of the cost value. At each iteration, the first entry is fetched and removed from the SSL. The point in this entry, $\mathbf{y}\left(t_{i}\right)$, is tracked using traditional HARP tracking but starting from $\mathbf{y}^{\prime}\left(t_{i+1}\right)$ instead of $\mathbf{y}\left(t_{i}\right)$. Because the actual location $\mathbf{y}\left(t_{i+1}\right)$ is close to $\mathbf{y}^{\prime}\left(t_{i+1}\right)$ (as discussed above), HARP tracking is highly likely to converge to the correct position. After that, those neighbors of $\mathbf{y}\left(t_{i}\right)$ that have not been tracked and are not already labeled as boundary points are inserted into the list based on their cost values. The estimated locations of newly inserted neighboring points at time $t_{i+1}$ are computed using (6).

It is important to track every point inside the tissue first before growing the region into non-tissue regions. This is because the HARP value is quite noisy in the air, blood, or bone, where no tag pattern can be found in the image. To encourage the region growing process to visit tissue points first, the cost function that we use to sort the SSL is defined as:

$$
c\left(\mathbf{y}\left(t_{i}\right)\right)=\sum_{i=1}^{2}\left|W\left(\alpha_{i}\left(\mathbf{y}\left(t_{i}\right), t_{i}\right)-\alpha_{i}\left(\mathbf{y}^{\prime}\left(t_{i+1}\right), t_{i+1}\right)\right)\right|
$$

Since the phase values inside the tissue are smooth, for a point inside the tissue the wrapped difference between its actual phase $\left(\alpha_{i}\left(\mathbf{y}\left(t_{i}\right), t_{i}\right)\right)$ and the phase at its estimated location is small, and the cost function has small value. Therefore the point is placed at the front of the SSL and is visited early.

\subsection{Implementation of HARP refinement algorithm}

This algorithm can be implemented as follows:

Track manually selected seed point(s) over all time frames For each time frame $t_{i}$

Find neighbors of the seed(s), and create the SSL

While the SSL is not empty

Remove the first node $\mathbf{x}\left(t_{i}\right)$ from SSL

Find $\mathbf{x}\left(t_{i+1}\right)$ using HARP tracking

Label $\mathbf{x}\left(t_{i}\right)$ as tracked point

For every neighbor $\mathbf{y}_{k}\left(t_{i}\right)$ of $\mathbf{x}\left(t_{i}\right)$

If not tracked point and not boundary point Calculate $\mathbf{y}_{k}^{\prime}\left(t_{i+1}\right)$, and the cost $c\left(\mathbf{y}\left(t_{i}\right)\right)$ Insert $\mathbf{y}_{k}\left(t_{i}\right)$ in the SSL based on $c\left(\mathbf{y}\left(t_{i}\right)\right)$ Label $\mathbf{y}_{k}\left(t_{i}\right)$ as boundary point

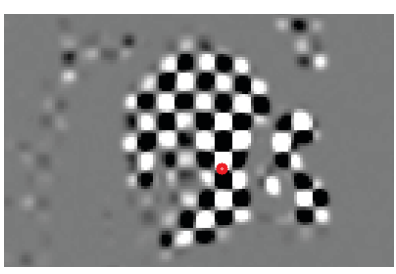

(a)

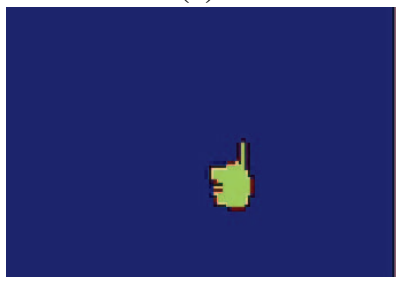

(c)

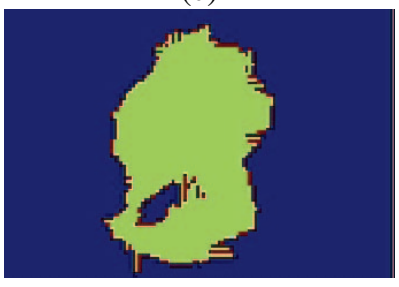

(e)

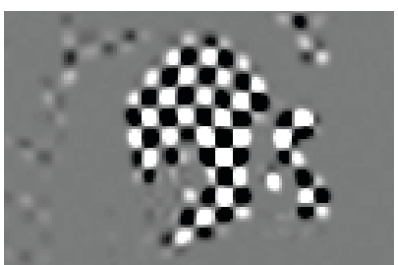

(b)

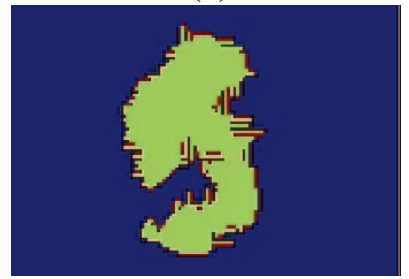

(d)

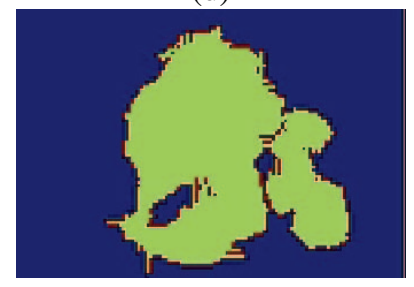

(f)
Fig. 3. Illustration of the region growing process. (a) is the checkboard image at the first time frame by overlaying the two tagged images with different orientations, (b) is the checkboard image at the second time frame. The red dot in (a) is the manually selected seed point. (c-f) shows how the region grows. The green color means tracked points, brown means boundary points, and blue means points that are not tracked and not boundary points.

\section{EXPERIMENT RESULTS}

Our method was applied on the tagged MR images of the tongue. The images were collected on a 1.5T Marconi scanner when the subject uttered "eeoo" repeatedly. The images were acquired in 12 time frames with a temporal resolution of $66 \mathrm{msec}$. The interpolated spatial resolution was $1.09 \mathrm{~mm}$ $\times 1.09 \mathrm{~mm} \times 7 \mathrm{~mm}$. Four sets of SPAMM images were collected: horizontal tagging with $\left[+90^{0}+90^{\circ}\right]$ and $\left[+90^{0}-90^{0}\right]$ , and vertical tagging with $\left[+90^{0}+90^{0}\right]$ and $\left[+90^{0}-90^{\circ}\right]$ tagging pulses. As preprocessing, the MICSR [9] images were reconstructed from these 4 sets of data. Our method was implemented in $\mathrm{C}$, and compiled in Matlab 7 (Mathworks, Natick MA). On a computer with Intel Core Duo $1.83 \mathrm{GHz}$ processor and 1.0G ram, our implementation took about $0.2 \mathrm{sec}-$ ond to track an 128 by 128 image for one time frame.

Fig. 3 shows the intermediate results of the region growing process on the mid-sagittal slice. Starting from the seed point, the points inside the tongue were tracked first. The outside points were tracked only after all points inside the tongue were tracked. In Fig. 4 a grid of points was placed 


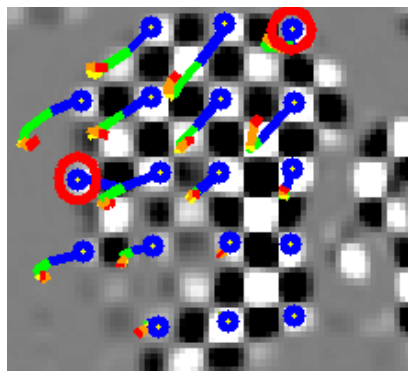

(a)

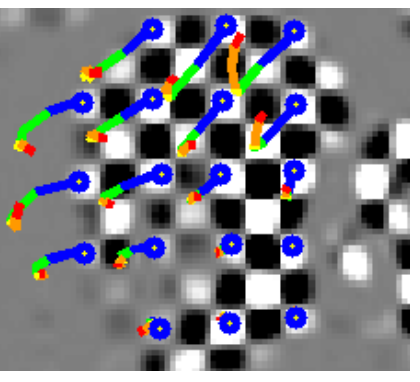

(b)
Fig. 4. The trajectory of some points on tagged MR image over 12 time frames. (a) is the results of traditional HARP tracking. The points in the circle were mistracked. (b) is the HARP refinement results.

inside the tongue and tracked. Some points were mistracked (Fig. 4(a)) when using the traditional HARP tracking, but correctly tracked using our HARP refinement method (Fig. 4(b)). Fig. 5 illustrates how our refinement method improves the Lagrangian strain calculation. The Lagrangian strain is computed as the length change of line segments with respect to the length at first time frame. It has a positive value when stretching and a negative value when contracting. In Fig. 5(b), the number 1 and number 4 line segments were mistracked in traditional HARP tracking, which made the Lagrangian strain calculation wrong (Fig. 5(d)). However, they are correctly tracked using the new HARP refinement method (Fig. 5(c) and (e)).

\section{CONCLUSION}

In this paper we presented a refinement method of HARP tracking based on seeded region growing. Experimental results show that this method can reliably track every point inside the tissue even in the case of large motion when the traditional HARP tracking fails. This method is also computationally fast and makes it feasible to compute Lagrangian strain between arbitrary points in real time.

\section{REFERENCES}

[1] N. F. Osman et al., "Cardiac motion tracking using CINE harmonic phase (HARP) magnetic resonance imaging," Mag. Res. Med., vol. 42, pp. 1048-1060, 1999.

[2] N. F. Osman and J. L. Prince, "Visualizing myocardial function using HARP MRI," Phys. Med. Biol., vol. 45, pp. 1665-1682, 2000.

[3] V. Parthasaranthy, M. NessAiver, and J. L. Prince, "Tracking tongue motion from tagged magnetic resonance images using harmonic phase MRI (HARP-MRI)," Proc. Of 15th Intl. Cong. of Phon. Sci., 2003.

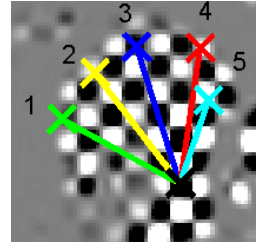

(a)

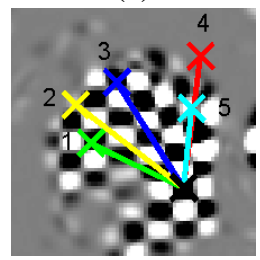

(b)

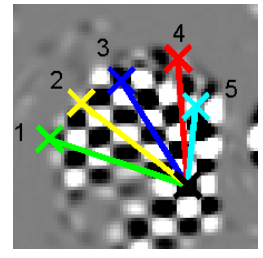

(c)

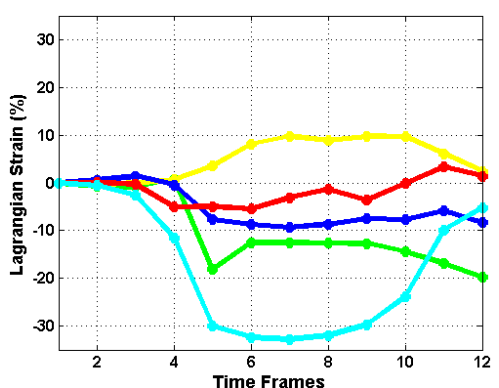

(d)

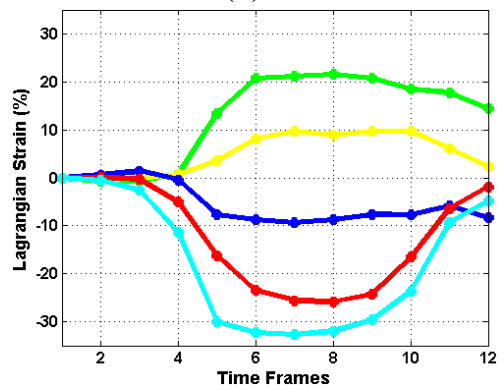

(e)
Fig. 5. The action of Genioglossus (GG). (a) shows five segments of GG at the first time frame. They are then tracked using both traditional HARP method and our HARP refinement method throughout all the time frames, The tracked position at the last time frame is shown in (b) and (c) respectively. (d) and (e) shows the Lagrangian strains of the 5 line segments change with time. (d) is the result of traditional HARP method, and (e) is the result of HARP refinement.

[4] X. Liu, M. Stone, and J. L. Prince, "Tracking tongue motion in three dimensions using tagged MR images," in IEEE Int. Sym. Biomed. Imag., 2006, pp. 1372-1375.

[5] W. S. Levine et al., "A nonlinear incompressible model of the human tongue," SIAM News, vol. 39, no. 2, 2006.

[6] A. M. Khalifa, A. M. Youssef, and N. F. Osman, "Improved harmonic phase (HARP) method for motion tracking a tagged cardiac MR images," in 2005 IEEE EMBS Ann. Conf., 2005, pp. 4298-4301.

[7] N. F. Osman, Measuring regional cardiac function using harmonic phase magnetic resonance imaging, Ph.D. thesis, The Johns Hopkins University, 2001.

[8] R. Adams and L. Bischof, "Seeded region growing," IEEE Trans. Pattern Anal. Mach. Intell., vol. 16, pp. 641647, 1994.

[9] M. NessAiver and J. L. Prince, "Magnitude image CSPAMM reconstruction (MICSR)," Mag. Res. Med., vol. 50, no. 2, pp. 331-342, 2003. 